\title{
On Negative Differential Mobility in Nanophotonic Device Functionality
}

\author{
Emmanuel A. Anagnostakis \\ Hellenic Air Force Academy, Dekeleia, Greece \\ E-mail: emmanagn@otenet.gr \\ Received August 19, 2011; revised September 17, 2011; accepted September 28, 2011
}

\begin{abstract}
A negative differential mobility (NDM) of the two-dimensional carrier-gas against some proper external regulator allowing for gradual controlled modification of the nanointerfacial environment tends to occur as interwoven with nanophotonic device functionality. In this work, several instances, in our two-decade principal research, of both experimental observation and conceptual prediction concerning nanophotonics NDM are reconsidered towards outlining a global potential for the appearance of the effect.
\end{abstract}

Keywords: Nanophotonics, Two-Dimensional Electron-Gas, Semiconductor-Device Nanointerface, Negative Differential Mobility, Optoelectronics Nanotechnology

\section{Introduction}

Photonics is underlain by absorption, emission, generation, handling, and exploiting light, typically within the electromagnetic radiation spectrum range between 100 $\mathrm{nm}$ of the ultraviolet and $1000 \mathrm{~nm}$ of the infrared-with the human visual perception focused upon the $400-700$ $\mathrm{nm}$ optical interval.

Nanophotonics, now, with its recently formulating disciplinary autonomy, traces, interprets, and envisions nanotstructural spatial confinement-induced, often mystifying, modifications in light propagation and light-matter interaction. Nanophotonics, thus, is studying the essence and manifestations of confined electron de Broglie waves and confined light-wave photon flows. Interestingly, between 370 and $1600 \mathrm{~nm}$ there function nanophotonic devices based on the GaN/GaAs, InAs/ GaAs and InAs/InP materials-systems, nowadays adequately reaching the middle infrared (mid-IR) regime.

Within the branch of electron-confinement Nanophotonics, a crucial feature permeating semiconductor nanodevice photonic functionality is the appearance of a negative differential mobility (NDM) of the two-dimensional carrier-gas against some proper external regulator (materialising as instantaneous cumulative photonic intake) allowing for gradual controlled modification of the nanointerfacial environment.

In this work, several instances [1-20], in our two-decade principal research, of both experimental observation and conceptual prediction concerning NDM are reconsidered towards outlining a global potential for the appearance of the effect, the essence of which consists in the variation of the nanointerfacial two-dimentional electron gas's mobility becoming negative against further positive proper regulatory agent's (bias's, photonic dose's) change for some regulatory agent's value-interval(s).

\section{NDM Occurrence in Nanophotonics Homodevices}

In Figure 1, the energy band depth-profile of a generichomointerface nanophotonics device is presented: The band bending occurs at the illuminated n-type upper semiconductor-layer's surface and within the nanodevice interface extending between the equilibrium parts of this epilayer and the relatively p-type-like lower semiconductor-layer. The nanointerface (NIF) potential-energy barrier $e U_{b}$ is equal to $e\left(U_{b i}-U_{p h}\right)$, where $U_{b i}$ is the NIF diodic built-in voltage and $U_{p h}$ is the generated evolving photovoltage $[2-4,7]$. The photocurrent density J through the NIF consists of the space-charge-region photogeneration-current density $\mathrm{J}_{\mathrm{g}}$, the current density $\mathrm{J}_{\mathrm{p}}(\mathrm{x})$ at the NIF upper-boundary locus $\mathrm{x}$ owing to hole diffusion from the n-type epilayer downwards, and the current density $\mathrm{J}_{\mathrm{n}}(\mathrm{x}+\mathrm{w})$ at the NIF lower-boundary locus $\mathrm{x}+\mathrm{w}$ owing to electron diffusion from the p-type sublayer upwards [1]. 


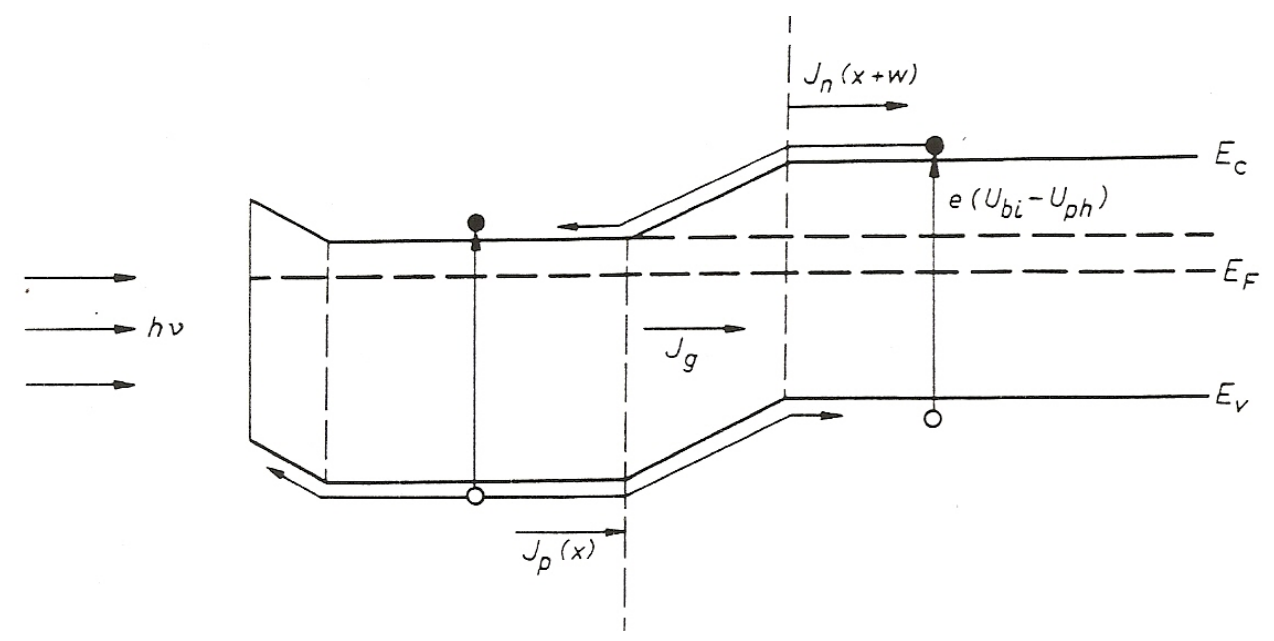

Figure 1. Energy band depth-profile of a generic-homointerface nanophotonics device: The nanointerface (NIF) potentialenergy barrier $e U_{b}$ is equal to $e\left(U_{b i}-U_{p h}\right)$, where $U_{b i}$ is the NIF diodic built-in voltage and $U_{p h}$ is the generated evolving photovoltage.

Experimental findings for the mean electron-mobility ( $\mu$ ) ( in $10^{2} \mathrm{~cm}^{2} /(\mathrm{V} \mathrm{s})$ ) within the n-type (Si-ion implantation) epilayer of representative photonics NIF-devices $[1,9,12]$ vs. incoming instantaneous cumulative photondose $\delta$ ( in photons $/ \mathrm{cm}^{2}$ ) have been discussed in previous works of ours: The functional feature of negative differential mobility (NDM) is being registered from the mobility peak (at total photonic intake $\delta_{\text {peak }}$ of around $1 \times$ $10^{11}$ photons $/ \mathrm{cm}^{2}$ ) to the mobility valley (at cumulative photon dose $\delta_{\text {valley }}$ of around $4.64 \times 10^{12}$ photons $/ \mathrm{cm}^{2}$ ). Each instantaneous value of the average mobility is determined within the respective instantaneous extension of the photo-widening flat-band portion of the epilayer through the experimentally measured (mean) sheet electron conductivity $(\sigma \mathrm{d})$ and (mean) sheet electron concentration (nd) of this flat-band epilayer extension by $\langle\mu\rangle=$ $(\sigma d) /(n d)$. The local mobility $\mu$, furthermore, at the instantaneous NIF-boundary locus $\mathrm{x}$ is interlplaying with the mean mobility $\langle\mu>$ and the dose-rates $u$ and $v$ of evolution of ( $\sigma \mathrm{d})$ and (nd), respectively, according to the causal correlation $[1,9,10,12]$ :

$$
\mathrm{v}=[\mathrm{u} /(\mathrm{e}<\mu>2)][2<\mu>-\mu],
$$

with e being the absolute value of the electron charge.

\section{NDM Occurrence in Nanophotonics Het- erodevices}

In Figure 2, the energy band depth-profile of a generic-heterointerface nanophotonics device is sketched: The front, wider-bandgap, n-type semiconductor-layer is succeeded by the lower, narrower-bandgap, relatively p-type-like one. The NIF, now, hosts the ionized-donor depletion zone, on the epilayer side, and the potentialenergy, approximately triangular, QW, formed within the sublayer's upper part. The photocurrent density J through the NIF comprises the current density $\mathrm{J}_{\mathrm{g}}$ produced by photogeneration within both the ionized-donor depletion-zone and the QW, the current density $\mathrm{J}_{\mathrm{p}}(\mathrm{x})$ at the NIF upper-boundary locus $\mathrm{x}$ owing to hole diffusion from the n-type epilayer downwards, and the current density $\mathrm{J}_{\mathrm{n}}(\mathrm{x}+\mathrm{w}+\mathrm{L})$ at the NIF lower-boundary (deeper by the consecutive extensions $\mathrm{w}$ and $\mathrm{L}$ of the depletion zone and the $\mathrm{QW}$ ) locus $\mathrm{x}+\mathrm{w}+\mathrm{L}$ owing to electron diffusion from the p-type sublayer upwards $[3,5,7,8]$. The onset of the occupancy of the first excited QWsubband (of bottom energy $E_{1}$ ) occurs as soon as the two-dimensional electron gas (2DEG) population already confined within the NIF QW energy-wise span the interval $\Delta \mathrm{E}=\mathrm{Z}_{0} / \rho$, from the $\mathrm{QW}$ fundamental-subband bottom onwards, with $Z_{0}$ constituting the capacity of the fundamental subband and $\rho$ being the parabolic-model two-dimensional density of states (with respect to energy graduation and QW-bottom cross-section interval). An effective harmonic oscillator simulating the prime functionality of the NIF QW leads to a simple, yet notionally adequate and rather universal, NIF descriptor $\Gamma=\mathrm{L} \Omega$, with $\Omega$ being the simulative-oscillator strength, shown to be expressible in terms of the carrier effective mass and the entailed-nanoheterojunction conduction- band discontinuity as $\Gamma=2\left(2 \Phi / \mathrm{m}^{*}\right)^{1 / 2}$.

The reduced (over dark value $\mu_{\mathrm{d}}$ ) persistent photoenhancement (PPE) $\left(\Delta \mu / \mu_{d}\right)$ in the 2DEG mobility $\mu$ has been experimentally registered vs. relative (with respect to a nanodevice-specific value $\delta^{*}$ ) total photonic intake $\left(\delta / \delta^{*}\right)$, for representative nanophotonics (modulationdoping) heterointerface-devices of previous studies of ours $[3,5,7,8,14,16,17]$ (based on a typical molecularbeam epitaxy $\mathrm{Si}: \mathrm{Al}_{0.3} \mathrm{Ga}_{0.7} \mathrm{As} / \mathrm{GaAs}$ nanoheterodiode 


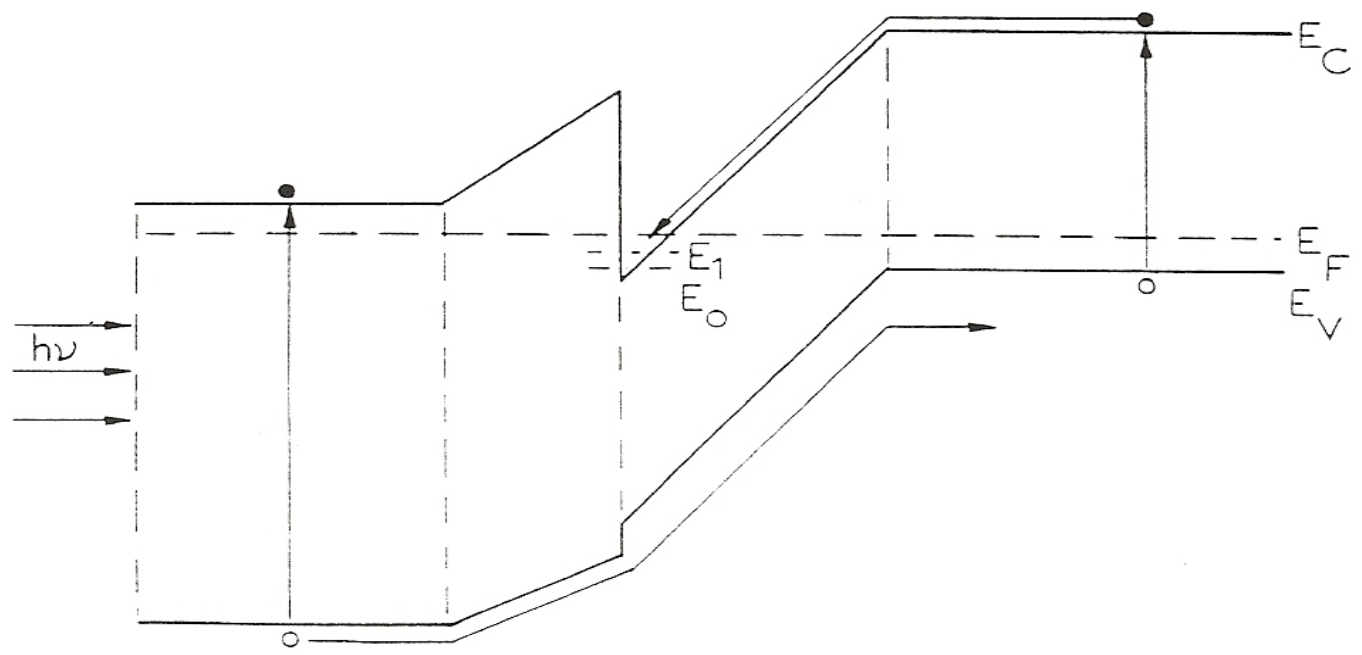

Figure 2. Energy band depth-profile of a generic-heterointerface nanophotonics device: The NIF, now, hosts the ionized-donor depletion zone, on the epilayer side, and the potential-energy, approximately triangular, QW, formed within the sublayer's upper part.

mounted upon a semi-insulating GaAs substrate): The NIF 2DEG mobility PPE $\Delta \mu$ is icreasing up to a critical relative cumulative photon-dose value $\left(\delta / \delta^{*}\right)_{\text {crit }}$ of the order of $4.79 \times 10^{2}$ and then it gets dropping, a NDM $([\mathrm{d}(\Delta \mu) / \mathrm{d} \delta]<0)$ regime being marked, tantamount to the process of occupancy of the first excited NIF QW subband, following the saturation of the capacity $Z_{0}=1.01 \times$ $10^{12}$ electrons $/ \mathrm{cm}^{2}$ (as determined through the experimentally traced photon-dose-evolution of the 2DEG sheet concentration $\zeta$, observed at the above critical total photonic intake) of the fundamental subband. The energy separation $\Delta \mathrm{E}$, now, between the nominal bottoms of these successive NIF QW subbands is (through the fundamental one's capacity) deduced to be of $35.3 \mathrm{meV}$, leading to a simulative harmonic oscillator's strength $\Omega$ of $5.354 \times 10^{13} \mathrm{~s}^{-1}$ yielding (through the NIF descriptor value $\Gamma=1.168 \times 10^{6} \mathrm{~m} / \mathrm{s}$ valid for the pertinent $\mathrm{QW}$ electron effective mass and conduction-band discontinuity) a 2DEG-QW effective spatial width $\mathrm{L}$ of $21.8 \mathrm{~nm}$.

\section{NDM Interweaving with Nanophotodevice Operational Principle}

In Figure 3., there appears the conduction-band depthprofile of the nanophotonics launcher-receptor quantum electron-device (LRD), proposed in 1994 [11] as a verticaltransport optoelectronic nanodevice relying upon resonant quantum-mechanical electron-tunnelling from a chargelauncher element (CLE) into a charge-receptor pocket (CRP) within an illuminated NIF semiconductor-heterostructure. In a model visualization, the LRD comprises an $\mathrm{Al}_{0.22} \mathrm{Ga}_{0.78}$. As epilayer hosting a narrow (of around 3 $\mathrm{nm}) \delta$-spike of high (especially with respect to background doping) sheet-density (on the order of $2.0 \times 10^{12}$

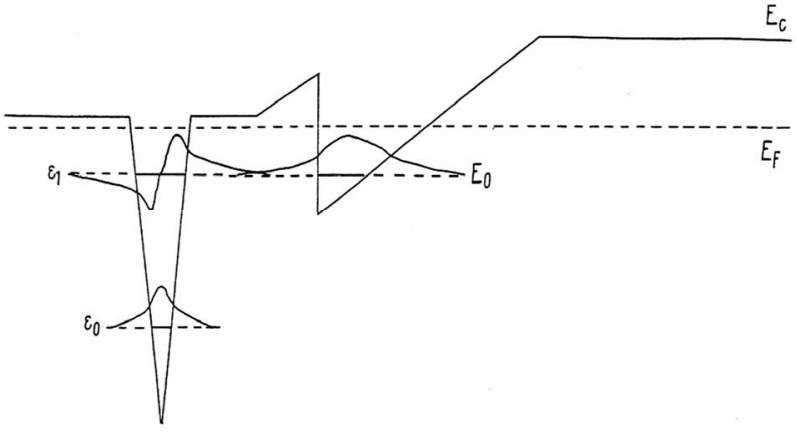

Figure 3. Conduction-band depth-profile of the nanophotonics launcher-receptor quantum electron-device (LRD), proposed in 1994 as a vertical-transport optoelectronic nanodevice relying upon resonant quantumme-chanical electron-tunnelling from a charge-launcher element (CLE) into a charge-receptor pocket (CRP) within an illuminated NIF semiconductor-heterostructure. Such an optoelectronic nanoswitching functionality would, then, be permeated by incidents of NDM owing to an appropriate timing for the energising of different individual effective-mobility values (via varying subband curvatures and scattering environments) characterising conductively interplaying subbands.

donors $/ \mathrm{cm}^{2}$ ) Si dopants as the photonic nanodevice CLE and a non-intentionally doped GaAs underlayer containing a nanoheterointerfacial 2DEG-QW (of spatial width on the order of $20 \mathrm{~nm}$, adjacent to the ionized-background-donor depletion-zone of the epilayer side) as the nanostructure CRP. A functionally strategic energy-matching between the CLE first excited sublevel $\varepsilon_{1}$ and the CRP fundamental sublevel $\mathrm{E}_{0}$ at around $50 \mathrm{meV}$ below the LRD Fermi level is attained, allowing for the possibility of resonant quantum-mechanical tunnelling of part of first-excited-subband CLE-electron population (present there after the saturation of the local fundamental sub- 
band's capacity, at a critical cumulative photonic intake during the persistent-photoenhancement experimental procedure) into the fundamental CRP-QW subband. Such an optoelectronic nanoswitching functionality would, then, be permeated by incidents of NDM owing to an appropriate timing for the energising of different individual effective-mobility values $[5,11,16,17,19]$ (via varying subband curvatures and scattering environments) characterising conductively interplaying subbands.

\section{NDM Tractability via Nanophotonics Wavefunction-Engineering}

In Figure 4, furthermore, the wavefunction-engineering of a THz-LASER nanophotonics device proposed in 2010 [18] is outlined: The optically pumped dual-resonant-tunnelling LASER-action unipolar-charge transport mechanism is simulatively energized for an indicative generic semiconductor nanoheterostructure based on the conventional $\mathrm{Al}_{\mathrm{x}} \mathrm{Ga}_{1-\mathrm{x}} \mathrm{As} / \mathrm{GaAs}$ material system.

In particular, two asymmetric in the spatial width and in the energetic barrier height, communicating through an intervening barrier layer, approximately rectangular quantum wells, both formulated within (different portions of) the GaAs semiconductor are employed: The front QW [F] of spatial width of $96^{\circ} \mathrm{A}$ and energy barrier height of $221 \mathrm{meV}$, contained between a surface $\mathrm{Al}_{0.3}$ $\mathrm{Ga}_{0.7}$ As slab and the inter-QW communication barrier layer, and the back QW [B] of growth axis extension $162^{\circ} \mathrm{A}$ and energy confinement hill of $204 \mathrm{meV}$, spanning the region between the inter-QW communication barrier layer and a botton $\mathrm{Al}_{0.33} \mathrm{Ga}_{0.67}$ As slab. The intervening, inter-QW communication barrier layer may non-exclusively be regarded as the succession (either abrupt or graded) of two rather equithick sublayers of $\mathrm{Al}_{0.3} \mathrm{GA}_{0.7}$ As and $\mathrm{Al}_{0.33} \mathrm{Ga}_{0.67}$ As. It is, then, algo-

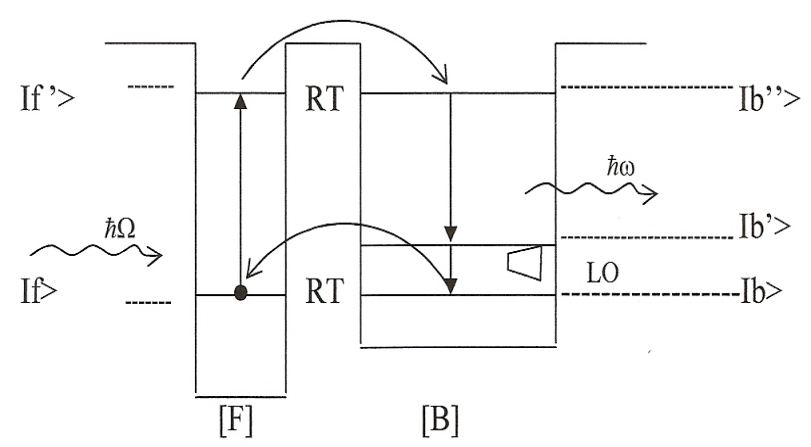

Figure 4. Wavefunction-engineering of a THz-LASER nanophotonics device proposed in 2010: The optically pumped dual-resonant-tunnelling LASER-action unipolar-chargetransport mechanism is simulatively energised for an indicative generic semiconductor nanoheterostructure based on the conventional $\mathrm{Al}_{\mathrm{x}} \mathrm{Ga}_{1-\mathrm{x}} \mathrm{As} / \mathrm{GaAs}$ material system. rithmically determined (through Quantum-Well Tridiagonal Algorithm [20]) that the partially localised conductivity electron eignestates accommodated by the couple of communicating QWs in the model application under study correspond to the energy eigenvalues (measured within each QW from its energetic bottom upwards): $\mathrm{E}$ (If $>$ ) $=32 \mathrm{meV}, \mathrm{E}$ (If '>) $=136 \mathrm{meV}$, for the front QW fundamental and first excited bound state, respectively, and $\mathrm{E}(\mathbf{I} \mathbf{b}>)=14 \mathrm{meV}, \mathrm{E}\left(\mathbf{I} \mathbf{b}^{\prime}>\right)=55 \mathrm{meV}$, and $\mathrm{E}(\mathbf{I} \mathbf{b}$ '> $)=121 \mathrm{meV}$ - for the back QW fundamental, first excited, and second excited bound state, respectively.

Notably, against this predicted energy eigenvalue configuration, the fundamental back QW eigenstate Ib> elevated by $14 \mathrm{meV}$ over the back QW energetic botton finds itself well aligned with the conjugate fundamental eigenstate If> of the front QW raised above its QW energetic botton by an amount corresponding to the inter QW energetic bottom discrepancy plus, about, the former fundamental eigenstate $\mathbf{I b}>$ height over its local QW bottom. In an analogous manner, the uppermost bound eigenstates of the two communicating QW, emerge aligned, as the difference in the height of each over its local QW bottom almost cancels the energetic height asymmetry of the two QW bottoms.

The determinable intersubband transition (ISBT) effective dipole lengths, furthermore, demonstrate the oscillator strengths supporting the different ISBT events, whereas the LASER action population inversion predicted would lead to the device stimulated optical gain.

The notional and functional framework, furthermore, of the principle of operation of such an intersubband THzLASER nanophotonics device provides fruitful ground [5,14-16] for the prediction, monitoring, and registration of NDM features marking incidents of local transition and resonant-tunnelling vertical transport, the essence of the innovation of a nanophotonics launcher-receptor quantum electron-device (LRD) [11] getting recapitulated and modellingly advanced and generalised.

\section{Conclusions}

Several instances, in our two-decade principal research, of both experimental observation and conceptual prediction concerning nanophotonics NDM have been reconsidered, the technological importance of the NDM crucial feature having been envisaged to be emerging.

There derives worth mentioning that a global potential for the manifestation of NDM would be the nanophotonic device's capacity for simultaneously supporting subsets of the interfacial conductivity-carrier ensemble through distinctly mobility-valued mechanisms. 


\section{References}

[1] E. A. Anagnostakis, "Characterisation of Semiconductor Epitaxial Layer Interfaces by Persistent Photoconductivity," Physica Status Solidi A, Vol. 126, No. 2, 1991, pp. 397-410. doi:10.1002/pssa.2211260211

[2] E. A. Anagnostakis, "Photoconductive Gain of Semiconductor Epitaxial Layers," Physica Status Solidi A, Vol. 127, No. 1, 1991, pp. 153-158. doi:10.1002/pssa.2211270116

[3] D. E. Theodorou and E. A. Anagnostakis, "Persistent Photoconductivity and DX Centres," Physical Review B, Vol. 44, 1991, pp. 352-3354. doi:10.1103/PhysRevB.44.3352

[4] E. A. Anagnostakis, "Photoconductive Response of GaAs Epitaxial Layers," Applied Physics A, Vol. 54, No. 1, 1992, pp. 68-71. doi:10.1007/BF00348133

[5] E. A. Anagnostakis, "Proposition of an Effective Wave Function for the 2DEG within MODFET Heterostructures," Physica Status Soilidi B, Vol. 171, No. 2, 1992, pp. K75-K78. doi:10.1002/pssb.2221710226

[6] E. A. Anagnostakis, "Lateral Photovoltage as a Probe of MODFET Channel Disorder," Physica Status Solidi B, Vol. 172, 1992, pp. K61-K63. doi: $10.1002 /$ pssb. 2221720228

[7] E. A. Anagnostakis, "Determination of Persistent Photoconductivity within Semiconductor Epitaxial Layers by Photoconductive Gain," Physical Review B, Vol. 46, No. 12, 1992, pp. 7593-7595. doi:10.1103/PhysRevB.46.7593

[8] E. A. Anagnostakis and D. E. Theodorou, "Determination of Two Dimensional Electron Gas Population Enhancement within Illuminated Semiconductor Heterostructures by Persistent Photoconductivity," Journal of Applied Physics, Vol. 73, No. 9, 1993, pp. 4550-4554. doi:10.1063/1.352800

[9] E. A. Anagnostakis, "Negative Differential Mobility Features in the Persistent Photoconductive Response of Semiconductor Devices," Physica Status Solidi A, Vol. 136, No. 1, 1993, pp. 247-250. doi:10.1002/pssa.2211360131

[10] E. A. Anagnostakis, "On a Generic Equation Modelling the Persistent Photoconductive Response of Semiconductor Devices," Physica Status Solidi B, Vol. 177, No. 2,
1993, pp. 533-536. doi:10.1002/pssb.2221770230

[11] E. A. Anagnostakis, "Designing a Launcher-Receptor Quantum Electron Device," Physica Status Solidi B, Vol. 181, No. 1, 1994, pp. K15-K17. doi: $10.1002 /$ pssb. 2221810127

[12] E. A. Anagnostakis, "Quantum Well Character of Semiconductor Homostructures Underlying the Occurrence of a Negative Differential Mobility," Physica Status Solidi A, Vol. 141, No. 2, 1994, pp. 373-379. doi:10.1002/pssa.2211410216

[13] E. A. Anagnostakis, "Photoconductive Studies of Carrier Mobility across Semiconductor Surface Depletion Zones," Physica Status Solidi A, Vol. 146, No. 2, 1994, pp. K9K11. doi:10.1002/pssa.2211460236

[14] E. A. Anagnostakis and D. E. Theodorou, "Semiconductor Heterointerface Characterisation via Effective Harmonic Oscillator Simulation," Physica Status Solidi B, Vol. 188, No. 2, 1995, pp. 689-695. doi: $10.1002 /$ pssb. 2221880212

[15] E. A. Anagnostakis, "Nanoheterointerface Wave Function Penetration Length Photonic Characterization," Reviews on Advanced Materials Science, Vol. 12, No. 2, 2006, pp. 182-188.

[16] E. A. Anagnostakis, "Semiconductor-Nanoheterointerface Eigenstate Photonic Modification", Journal of Non-Crystalline Solids, Vol. 354, No. 1, 2008, pp. 4233-4237. doi:10.1016/j.jnoncrysol.2008.06.029

[17] E. A. Anagnostakis, "Responsivity Robustness of Radioctivity-Irradiated Nanosensors," WSEAS Transactions on Circuits and Systems, Vol. 8, No. 3, 2009, pp. 311-320.

[18] E. A. Anagnostakis, "On a Scheme of Nanoheterointerfacial Intersubband 15-THz Luminescence," Physica B, Vol. 405, No. 1, 2010, pp. 25-28. doi:10.1016/j.physb.2009.08.010

[19] E. A. Anagnostakis, "Optoelectronic Nanoheterointerface Functional Eigenstate Photodynamics," Physica B, Vol. 405, No. 1, 2010, pp. 38-40. doi:10.1016/j.physb.2009.08.002

[20] E. A. Anagnostakis, "Quantum-Well Tridiagonal: A Qualitative Comprehension of Optoelectronics Nanotechnology," WSEAS Transactions on Circuits and Systems, Vol. 9, No. 1, 2010, pp. 1-10. 